\title{
2 U NECESIDADES BÁSICAS DE APRENDIZAJE DE LAS PERSONAS ADULTAS EN EL MARCO DEL PROGRAMA DE LA EDUCACIÓN PARA TODOS: DE JOMTIEN A DAKAR
}

Florentino Sanz Fernández

Universidad Nacional de Educación a Distancia

\section{DECLARACIÓN DE LOS DERECHOS HUMANOS Y EDUCACIÓN DE ADULTOS}

El objetivo de generalizar la educación a toda la población mundial viene siendo propuesto por la UNESCO desde la firma de la Declaración de los derechos humanos. En su artículo 26, se dice que "toda persona tiene derecho a la educación. La educación debe ser gratuita, al menos en lo que se refiere a la enseñanza elemental y fundamental. La enseñanza elemental es obligatoria"'.

Los dos términos claves que aparecen en este texto son el de enseñanza elemental y el de enseñanza fundamental. Enseñanza elemental en los trabajos preparatorios de la redacción venía a significar una instrucción mínima y se acercaba más al concepto de enseñanza escolar primaria de niños y adolescentes. La educación fundamental, que también se tradujo por educación de base, apuntaba a la educación de adultos. Para proclamar el derecho de todos, niños y adul-

\footnotetext{
${ }^{1}$ Declaración Universal de Derechos humanos adoptada y proclamada por la Asamblea general de las Naciones Unidas el 10 de diciembre de 1948.
} 
tos, a la educación se incluyeron los dos términos en la redacción definitiva de la Declaración. Pero el término fundamental seguía induciendo a una doble interpretación. Implicaba la idea de cimiento y de posibilidad abierta a un futuro (lo que después sería aprender a aprender o aprender a lo largo de toda la vida) y la idea de apertura a un contexto comunitario (aprender a satisfacer las necesidades más inmediatas de una determinada comunidad en sanidad, agricultura, higiene, etc.) convirtiéndose en sinónimo de educación comunitaria y acercándose en cierta manera al concepto de educación mínima por el hecho de reducir su objetivo a satisfacer los aprendizajes inmediatamente necesarios, sin poner tanto el acento en los aprendizajes necesarios a medio y largo plazo. Dada la riqueza de matices que iba saliendo en la elaboración del texto, el término de educación fundamental se utilizó más para significar la educación que posibilita seguir aprendiendo y el término de educación básica para designar la educación que posibilita actuar inmediatamente en una determinada comunidad. Término muy cercano, por otra parte, al de educación mínima o suficiente que ha caracterizado negativamente a la imagen de educación de adultos.

La introducción del término educación fundamental en la Declaración de los Derechos Humanos implicaba, en medio de todas aquellas matizadas discusiones, una voluntad clara de extender el derecho de la educación no sólo a los niños sino también a los adultos. Una concepción de adultos abierta no sólo a la comunidad, al corto plazo, al mínimo exigible por una situación dada, sino al futuro y a la construcción indefinida de aprendizajes. Se distingue, además, entre educación elemental y fundamental para no reducir la educación de adultos a la educación escolar primaria. Lógicamente todos estos matices no aparecen formulados expresamente en el artículo 26 de la declaración de los derechos humanos, pero un análisis detenido de las comisiones preparatorias de la redacción del texto conduce fácilmente a estas conclusiones ${ }^{2}$.

\section{DIFERENTES ESTRATEGIAS SOBRE EDUCACIÓN FUNDAMENTAL EN LOS AÑOS CINCUENTA: DESDE APRENDER A LEER Y ESCRIBIR HASTA SATISFACER NECESIDADES FUNDAMENTALES DE APRENDIZAJE}

Una vez declarado el derecho de toda persona a la educación (no sólo de todos los niños sino también de los adultos) se establecen las estrategias concretas para abordar el desafío de universalizar la educación. Se distingue

\footnotetext{
${ }^{2}$ Para una mayor información sobre el usos de la diferente terminología utilizada en la redacción del artículo 26 de la Declaración de los Derechos Humanos se puede consultar: Le droit a l'éducation. Vers l'éducation pour tous, tout au long de la vie. En Rapport mondial sur l'éducation 2000. Anexe I Editions Unesco.
} 
entre objetivos de la educación fundamental (aprender a pensar y comunicar el pensamiento, adquirir técnicas profesionales, conocimiento del medio físico, conocimiento del medio humano individual y social, desarrollo de capacidades que permitan vivir en el mundo moderno, etc.) y estrategias o medios para conseguir dichos objetivos (campañas para aprender a leer y a escribir, enseñanza de agricultura y de higiene, desarrollo de la enseñanza primaria, extensión de la educación fundamental aparte de la secundaria, hogares culturales, información masiva mediante la radio, la prensa, medios impresos, etc.). Lo más fácil, en una primera etapa, fue centrarse en la estrategia de universalizar el aprendizaje de la lectura y la escritura, de tal forma que educación de adultos comenzó a identificarse con la lucha contra el analfabetismo y con la extensión y generalización de las más elementales habilidades académicas. Alfabetizar era una estrategia concreta y clara sobre la que se disponían datos cuantitativos. Los datos cuantitativos sobre analfabetismo se convirtieron en el único referente para medir las necesidades y las respuestas educativas en las diferentes sociedades ${ }^{3}$. Por otra, en los años 50 y 60 , muchos países que recobraron la independencia se propusieron la alfabetización como objetivo prioritario nacional.

En el caso de las personas mayores de 15 años, los analfabetos sumaban entonces un total de unos 700 millones. Las conferencias de Bombay (1952), El Cairo (1955), Lima (1956), Karachi (1960) y Abdis Abeba (1961) abordaron este tema mediante la programación de grandes campañas de alfabetización para los adultos ${ }^{4}$, y los distintos gobiernos aceleraron la posibilidad del acceso a la escuela para los niños y niñas con la esperanza de que llegaran alfabetizados a la edad adulta y atajar desde la niñez el objetivo de universalizar la alfabetización adulta para finales de los años ochenta. Los resultados, sin embargo, no fueron los esperados y se demostró que la estrategia utilizada no respondía a las necesidades fundamentales de aprendizaje por varias razones. En primer lugar, no se adaptaba al crecimiento demográfico. Es verdad que la alfabetización avanzaba, pero no al mismo ritmo que el crecimiento de la población, con lo que el número de analfabetos permanecía prácticamente inalterado o en aumento. En segundo lugar la habilidad lecto-escritora no era suficiente para cubrir las necesidades de aprendizaje y aparecían la necesidad de completar esa habilidad con otras habilidades sociales.

${ }^{3}$ Todavía una de las críticas que se ha hecho en el foro de Dakar es que los indicadores que miden la situación de educación de adultos sean fundamentalmente escolares. Se sabe que los indicadores escolares no miden bien porque miden el acceso a la escuela, pero no los resultados, y además no sólo las habilidades escolares cubren todas las necesidades de aprendizaje. La OCDE, teniendo en cuenta los resultados escolares, calcula que en los países desarrollados el analfabetismo funcional (iletrismo en Francia) se sitúa en torno a un $20 \%$. Si este es el caso de los países desarrollados no puede ser inferior o parecido en los países en desarrollo.

${ }^{4}$ Las experiencias de estas campañas nacionales habían sido positivas en los años treinta. En la URSS la proporción de analfabetos había descendido al 4,9\% en los hombres y al $16 \%$ en mujeres. 
Ante esta sospecha fundada, a mediados de los años sesenta se comienza un cambio de estrategia y se centra la atención en la calidad, la motivación de los adultos participantes en los programas, el uso real de los aprendizajes y, por lo tanto, el interés del aprendizaje ligados a procesos de desarrollo económico y de participación ciudadana. Son los años en que la educación de adultos manifiesta una doble utilidad: como factor del crecimiento económico por una parte y como elemento de liberación política por otra. Aprender dejaba de ser un valor exclusivamente académico para convertirse en un valor económico y político que habría de servir para vivir mejor, para ser más libres y más autónomos. Alfabetizar tenía un valor funcional de tipo profesional que potenciaría el crecimiento económico de muchos países en desarrollo (capital humano) y también una funcionalidad política que potenciaría la democratización real de la sociedad (liberación de Paulo Freire). Son los años en que la Conferencia mundial de ministros de educación en Teherán, septiembre de 1965, posibilitaría la puesta en marcha del Programa experimental mundial de alfabetización (PEMA) lanzado en 1966 por la UNESCO y el PNUD y que puso en interacción procesos educativos y procesos de desarrollo. Años que coinciden, como decimos, con los programas alfabetizadores de Paulo Freire, para quien aprender a leer y a escribir no era solamente una actividad escolar, ni se relacionaba solamente con la actividad profesional y el empleo, sino que era sobre todo un acto político. Se constata así, a finales de los ochenta, que saber leer y escribir es solamente un aspecto más de las necesidades de aprendizaje de los adultos. Sin negar la importancia de la alfabetización ni la de la escuela, se comprende cada vez mejor que no se puede reducir la enseñanza fundamental a la adquisición de las tradicionales habilidades académicas y comienza a hablarse de la necesidad de adquirir habilidades sociales. Surge así una tercera etapa a finales de los años ochenta en la educación de personas adultas (Conferencia de Jomtien) que tiende a afrontar el conjunto de necesidades fundamentales de aprendizaje de distinta manera.

La tercera estrategia sobre la educación fundamental que comienza a programarse en Jomtien plantea una visión ampliada y más compleja de la alfabetización, que supera el esquema académico y que lo plantea como una estrategia intersectorial en la que no solamente intervienen los educadores sino también los políticos y economistas; y en la que no importa solamente el aprendizaje sino las condiciones de su efectividad.

\section{LAS APORTACIONES DE LA "EDUCACIÓN PARA TODOS (EPT)" EN JOMTIEN A "LA EDUCACIÓN DE PERSONAS ADULTAS"}

Nos interesa detenernos especialmente en las aportaciones que, a nuestro juicio, pueden considerarse fundamentales para optimizar la práctica y el paradigma teórico de la educación de personas adultas: 
- Por una parte, el concepto de necesidades fundamentales de aprendizaje o necesidades básicas de aprendizaje para todos que supera al concepto tradicional de alfabetización.

- Por otra, el concepto de "visión ampliada de la educación básica" que, a nuestro juicio, sin ser exclusiva de la educación de adultos, orienta hacia una buena práctica educativa de las personas adultas.

\subsection{EN JOMTIEN SE SUPERA EL CONCEPTO TRADICIONAL DE ALFABETIZACIÓN}

"Los programas de alfabetización son indispensables dado que saber leer y escribir constituye una capacidad necesaria y es la base de otras aptitudes vitales" (art. 5).

Sin embargo los aprendizajes básicos que una persona necesita no sólo para sobrevivir sino para vivir con dignidad humana "abarcan tanto las herramientas esenciales para el aprendizaje (como la lectura y la escritura, la expresión oral, el cálculo y solución de problemas) como los contenidos básicos del aprendizaje (conocimientos teóricos y prácticos, valores y actitudes) necesarios para poder sobrevivir, desarrollar plenamente sus capacidades, vivir y trabajar con dignidad, participar plenamente en el desarrollo, mejorar la calidad de vida, tomar decisiones fundamentadas y continuar aprendiendo. La amplitud de las necesidades básicas de aprendizaje y la manera de satisfacerlas varian según cada país y cada cultura y cambian inevitablemente con el transcurso del tiempo" (Declaración art. 1.1.).

\subsubsection{El analfabetismo deja de ser considerado como una patología social, cuya responsabilidad está localizada en la escuela, para ser comprendido como un fenómeno estructural cuya responsabilidad es social}

El analfabetismo no ha de comprenderse como un problema exclusivamente académico, sino prioritariamente como un problema social que se ataja no sólo con estrategias micropedagógicas o intraeducativas (mejores textos, mejores profesores, más tiempo dedicado a la enseñanza, más recursos económicos para educación, etc.), sino con estrategias sociales macropedagógicas o extraeducativas (más puestos de trabajo, más tiempo libre, relaciones sociales más igualitarias, más perspectivas para desarrollar los aprendizajes, mejores condiciones de salud, etc.). La tarea de la alfabetización deja de ser sólo una técnica de trabajo académico, cuya eficacia depende de los profesionales de la educación, para convertirse además en una estrategia intersectorial de educadores, 
políticos y economistas cuya eficacia depende del diseño de sociedad que se quiere construir y del plan estratégico que se establece para conseguirlo.

\subsubsection{Por eso la alfabetización escolar empieza a dejar de ser considerada como la panacea para la solución de los problemas de desarrollo}

La alfabetización sólo favorece al desarrollo si, además de aumentar la escolarización a un ritmo anual de 10 millones, como por ejemplo se viene haciendo en esta década, aumenta también el dinero dedicado al desarrollo y no decrece, como paradójicamente viene ocurriendo también en esta misma década. El problema de la alfabetización no se resuelve posibilitando aprendizajes desde la pedagogía, sino posibilitando la efectividad de esos aprendizajes desde otros sectores como la economía o la política.

No puede justificarse que a más educación básica sucede automáticamente más bienestar, cuando a nivel global coinciden en un mismo periodo de tiempo dos fenómenos al parecer tan contradictorios como el aumento de recursos intraeducativos dedicados a la enseñanza y el aumento de pobres en el mundo. Por ejemplo, la década de los noventa ha sido la que más igualdad académica ha creado, multiplicando el acceso a la primera enseñanza, y también ha sido la década en la que más han aumentado las diferencias y desigualdades sociales. Diferencias y desigualdades que, a su vez, reproducen o reduplican más el complejo de la inutilidad de la enseñanza. Lo que se adelanta por un lado se estropea por otro.

En lugar de plantear solamente la universalización de la educación como medio para alcanzar la generalización del bienestar se hace necesario plantear, además y simultáneamente, una estrategia diferente que incluya la generalización del bienestar y del reparto de poder como medio para generalizar la educación fundamental.

\subsubsection{La alfabetización deja de tener un sentido estable y recobra dinamicidad}

Se reconoce que el nivel alfabetizador crece constantemente en nuestras sociedades. El concepto de educación fundamental ha evolucionado desde la habilidad lecto-escritora, pasando por las habilidades académicas de la enseñanza escolar primaria, para incluir ya, en muchos países, parte de la secundaria, etc. Cada reforma educativa eleva el nivel de exigencias fundamentales de aprendizaje.

Ha dejado, además, de estar asociada a los instrumentos convencionales del papel y el lápiz para referirse a las tecnologías digitales y dialógicas como 
saber interpretar mensajes que llegan por distintos cauces, saber escuchar y observar, saber trabajar la información, etc.

No bastará con saber leer o contar, si se quiere conservar el planeta, poner fin a los conflictos, acabar con la epidemia del sida y garantizar a todo el mundo una existencia digna (Víctor Ordóñez, portavoz de la conferencia de Dakar 2000: 3).

\subsubsection{Alfabetizar no es capacitar sino formar}

Se deja de entender la alfabetización como el desarrollo de capacidades aisladas para entenderla como desarrollo de capacidades relacionadas. Estar alfabetizado ya no hace referencia solamente al desarrollo de una capacidad aislada, como la de contar o escribir (alfabetización como la capacidad de comprender un texto impreso o comunicarse por escrito), sino que supone el desarrollo de varias capacidades relacionadas que permitan analizar, dialogar, comprender, valorar, escuchar, actuar, y, en definitiva, transformar aquella realidad a la que se refiere el texto.

En Jomtien se diferencia entre alfabetización y satisfacer necesidades básicas de aprendizaje. Para definir el analfabetismo hay que referirse a la vez a las competencias escolares tradicionales y a las competencias sociales para saber gestionar tareas en contextos extra escolares. Es verdad que una de las necesidades fundamentales que la gente necesita es la de saber leer y escribir, sobre todo en una sociedad letrada como la nuestra, pero ésta es una entre otras necesidades básicas.

Un resumen de esta nueva visión de la alfabetización fue presentado en la conferencia de Dakar por la División de la Educación Básica de la Unesco en el siguiente cuadro.

\begin{tabular}{|l|l|}
\hline \multicolumn{2}{|c|}{ Alfabetización para todos: una visión renovada } \\
\hline \multicolumn{1}{|c|}{ ANTES } & \multicolumn{1}{|c|}{ HOY } \\
\hline $\begin{array}{l}\text { El analfabetismo como patología social } \\
\text { y responsabilidad individual. }\end{array}$ & $\begin{array}{l}\text { El analfabetismo como fenómeno } \\
\text { estructural y responsabilidad social. }\end{array}$ \\
\hline $\begin{array}{l}\text { La alfabetización como la panacea para } \\
\text { el desarrollo y el cambio social. }\end{array}$ & $\begin{array}{l}\text { La alfabetización en el contexto de } \\
\text { intervenciones educativas y socio-eco- } \\
\text { nómicas más amplias. }\end{array}$ \\
\hline $\begin{array}{l}\text { La meta es "erradicar el analfabetis- } \\
\text { mo"," "bajar los índices de analfabetis- } \\
\text { mo", etc. }\end{array}$ & $\begin{array}{l}\text { La meta es crear ambientes y socieda- } \\
\text { des letradas. }\end{array}$ \\
\hline $\begin{array}{l}\text { La alfabetización asociada únicamente } \\
\text { con jóvenes y personas adultas. }\end{array}$ & $\begin{array}{l}\text { La alfabetización asociada con la infan- } \\
\text { cia, la juventud y la edad adulta. }\end{array}$ \\
\hline
\end{tabular}




\section{Alfabetización para todos: una visión renovada (Continuación)}

\begin{tabular}{|c|c|}
\hline ANTES & HOY \\
\hline $\begin{array}{l}\text { La alfabetización asociada con grupos } \\
\text { al margen de la escuela y con progra- } \\
\text { mas no-formales. }\end{array}$ & $\begin{array}{l}\text { La alfabetización como un proceso de } \\
\text { aprendizaje que tiene lugar tanto den- } \\
\text { tro como fuera de la escuela. }\end{array}$ \\
\hline $\begin{array}{l}\text { La alfabetización infantil y la alfabeti- } \\
\text { zación de jóvenes y adultos vistas y } \\
\text { desarrolladas como campos separados. }\end{array}$ & $\begin{array}{l}\text { La alfabetización infantil y la alfabeti- } \\
\text { zación de jóvenes y adultos articuladas } \\
\text { dentro de un marco y una estrategia } \\
\text { integradas de política. }\end{array}$ \\
\hline $\begin{array}{l}\text { La alfabetización centrada en el punto } \\
\text { de vista de la enseñanza. }\end{array}$ & $\begin{array}{l}\text { La alfabetización centrada en el punto } \\
\text { de vista del aprendizaje. }\end{array}$ \\
\hline $\begin{array}{l}\text { La alfabetización entendida como el } \\
\text { logro de un nivel inicial, básico, ele- } \\
\text { mental. }\end{array}$ & $\begin{array}{l}\text { La alfabetización entendida como alfa- } \\
\text { betización funcional (la alfabetización, } \\
\text { para ser llamada tal, debe ser significa- } \\
\text { tiva y funcional). }\end{array}$ \\
\hline $\begin{array}{l}\text { La meta aspira a la adquisición de la } \\
\text { alfabetización. }\end{array}$ & $\begin{array}{l}\text { La meta incluye la adquisición, el desa- } \\
\text { rrollo y el uso efectivo de la alfabetización. }\end{array}$ \\
\hline $\begin{array}{l}\text { La alfabetización separada de la educa- } \\
\text { ción básica ("alfabetización y educa- } \\
\text { ción básica"). }\end{array}$ & $\begin{array}{l}\text { La alfabetización vista como un compo- } \\
\text { nente integral de la educación básica. }\end{array}$ \\
\hline $\begin{array}{l}\text { La adquisición y el desarrollo de la alfa- } \\
\text { betización asociados con un período } \\
\text { específico en la vida de una persona. }\end{array}$ & $\begin{array}{l}\text { La alfabetización entendida como un } \\
\text { proceso de aprendizaje que dura y se } \\
\text { perfecciona a lo largo de toda la vida. }\end{array}$ \\
\hline $\begin{array}{l}\text { La adquisición de la alfabetización } \\
\text { como meta del primer grado o de los dos } \\
\text { primeros grados de la escuela primaria. }\end{array}$ & $\begin{array}{l}\text { La adquisición de la alfabetización } \\
\text { como una meta al menos de toda la } \\
\text { educación primaria. }\end{array}$ \\
\hline $\begin{array}{l}\text { La alfabetización asociada únicamente } \\
\text { con el lenguaje escrito y los medios } \\
\text { impresos. }\end{array}$ & $\begin{array}{l}\text { La alfabetización entendida como desa- } \\
\text { rrollo de la expresión y la comunica- } \\
\text { ción tanto oral como escrita, con una } \\
\text { visión del lenguaje como totalidad } \\
\text { (hablar, escuchar, leer, escribir). }\end{array}$ \\
\hline $\begin{array}{l}\text { Búsqueda de El método de alfabetización } \\
\text { válido en general y para todos los casos. }\end{array}$ & $\begin{array}{l}\text { Comprensión de que no existe un méto- } \\
\text { do único o universal de alfabetización. }\end{array}$ \\
\hline $\begin{array}{l}\text { La alfabetización como un área especí- } \\
\text { fica del currículo escolar (la asignatura } \\
\text { Lenguaje). }\end{array}$ & $\begin{array}{l}\text { La alfabetización a lo ancho del currí- } \\
\text { culo escolar. }\end{array}$ \\
\hline $\begin{array}{l}\text { La alfabetización asociada sólo con ins- } \\
\text { trumentos convencionales (típicamen- } \\
\text { te, papel y lápiz). }\end{array}$ & $\begin{array}{l}\text { La alfabetización asociada con instru- } \\
\text { mentos convencionales, pero también } \\
\text { con instrumentos modernos (papel y } \\
\text { lápiz, teclado y tecnologías digitales, etc). }\end{array}$ \\
\hline $\begin{array}{l}\text { La alfabetización como responsabili- } \\
\text { dad únicamente del Estado o única- } \\
\text { mente de la sociedad civil. }\end{array}$ & $\begin{array}{l}\text { La alfabetización como responsabili- } \\
\text { dad conjunta del Estado y la sociedad } \\
\text { civil. }\end{array}$ \\
\hline
\end{tabular}

Fuente: Borrador del documento de la sesión sobre Alfabetización para todos: una visión renovada para un plan decenal de acción. Participación de la División de educación básica de la UNESCO en Dakar. 


\subsection{EL CONCEPTO DE VISIÓN AMPLIADA DE LA EDUCACIÓN EN JOMTIEN}

Satisfacer las necesidades básicas de aprendizaje exige algo más que una renovación de compromiso con la educación básica en su estado actual. Lo que se requiere es una "visión ampliada" que vaya más allá de los recursos actuales, las estructuras institucionales, los planes de estudios y los sistemas tradicionales de instrucción, tomando como base lo mejor de las prácticas en uso (art. 2.1.).

Entre los elementos que se utilizan para describir esta "visión ampliada" de la educación, y que más tienen que ver con una visión también renovada de la práctica educativa de las personas adultas, destacamos los siguientes ${ }^{5}$ :

\subsubsection{La educación básica afecta a todos}

La educación básica debe proporcionarse a todos: niños, jóvenes y adultos. Con tal fin habría que extender los servicios educativos de calidad y tomar medidas consecuentes para reducir desigualdades (art. 3).

Las necesidades básicas de aprendizaje de los adultos y los niños deben atenderse alli donde existan. Los países menos adelantados y con bajos ingresos tienen necesidades particulares a las que se debe conceder prioridad en el apoyo internacional a la educación básica durante el decenio 1990 (art. 10.3).

La educación en el marco de Jomtien significa:

- Educación para todos independientemente de la edad o el género, y no sólo para los niños y niñas en edad infantil y de escolarización obligatoria.

- No sólo para los ciudadanos de los países en desarrollo. También en los países desarrollados hay personas con necesidades básicas.

- No sólo para marginados y excluidos. Muchas personas normales e integradas en la sociedad necesitan el desarrollo de capacidades básicas para proseguir determinados caminos que el mundo cambiante en el que vivimos les exige andar.

${ }^{5}$ Entre los análisis que se han hecho sobre el concepto de educación ampliada en general merece un interés especial el de R.M. Torres (2000), especialista que mantiene una participación destacada tanto en Jomtien como en Dakar. Nosotros fijamos el análisis en aquellos aspectos más directamente relacionados con la educación de personas adultas. 
- No sólo para analfabetos. Las personas analfabetas no son las únicas personas que tienen necesidades fundamentales de aprendizaje sin cubrir.

- Efectivamente hay que tomar medidas para reducir desigualdades, pero estas medida han de tomarse de tal forma que no discriminen aún más. Ha de hacerse de tal forma que no se reduzca sistemáticamente la educación básica a una acción compensadora de oportunidades perdidas, reparadora de daños ocasionados, adaptadora a situaciones establecidas que trate sistemáticamente a los educandos como "enfermos" o "inferiores" e inadaptados y no reconozca el valor de los aprendizajes que ya tienen independientemente de dónde, cómo y cuándo los haya adquirido.

\subsubsection{Educación básica para emprender y no sólo para aprender}

La educación básica, al ser un concepto dinámico y variable en el tiempo y en el espacio, no afecta solamente a poblaciones "marginadas" ni implica sólo compensar deficiencias del pasado. Necesitar formación básica en una sociedad tan dinámica como la nuestra afecta a muchas personas "normales" que, habiendo adquirido la educación básica que se les exigía en su tiempo de escolaridad obligatoria, se sienten, sin embargo, con problemas para participar activamente en la construcción social. También aquellas personas que, habiendo reducido su formación a la adquisición de habilidades estrictamente académicas, permanecen pobres en habilidades sociales, sentirán la necesidad de ampliar su formación para salir adelante en una sociedad tan dinámica como la que vivimos. La concepción de educación básica para todos ofrece una visión más positiva en el sentido de que no contempla al que la demanda solamente desde la perspectiva del pasado y desde los déficit escolares que tiene acumulados, sino desde la perspectiva del futuro y de los desafíos que la evolución social plantea. En este sentido la educación básica hace relación a aprender y a emprender.

\subsubsection{Educación básica no es lo mismo que la educación escolar primaria}

Los mismos textos de la declaración son ilustrativos por sí mismos:

El aprendizaje comienza con el nacimiento. Ello exige el cuidado temprano y la educación inicial de la infancia, lo que puede conseguirse mediante medidas destinadas a la familia, la comunidad o las instituciones, según convenga.

El principal sistema para impartir la educación básica fuera de la familia es la escuela primaria. Otros programas alternativos pueden ayudar a extender las necesidades de aprendizaje. 
Las necesidades básicas de aprendizaje de jóvenes y adultos son diversas y pueden satisfacerse mediante sistemas variados (art. 5).

La declaración universal no contemplaba un ciclo o un nivel particular de los sistemas educativos formales existentes entonces en el mundo como sinónimo de educación básica. Es más, se daba por supuesto que el nivel primario estaba en revisión tanto en los países en vías de desarrollo como en los desarrollados. En los países desarrollados se extendía la formación básica más allá del nivel primario hasta la secundaria. En Bélgica, Canadá, Finlandia, República Federal Alemana, Suiza y la antigua URS la escolaridad obligatoria ya se prolongaba hasta algún ciclo de enseñanza secundaria. En los países en vías de desarrollo el nivel primario escolar no se acomodaba del todo a las necesidades de algunos colectivos de zonas rurales o se la consideraba incapaz de satisfacer las necesidades de toda la población, ya que la tasa de escolarizados aumentaba, pero las cifras absolutas de no escolarizados también crecían. Esto explica en parte que, junto a los reformadores de los sistemas escolares de los países desarrollados que alargaban hasta la secundaria el nivel de educación considerado básico y obligatorio, en los países en desarrollo aparecieran movimientos críticos que demandaban modelos educativos desescolarizados o que buscaran formas de educación no escolar para poder satisfacer las necesidades educativas básicas de todos que no satisfacía la escolaridad primaria.

\subsubsection{La educación básica no es la educación mínima}

La educación básica es considerada, en esta visión ampliada de Jomtien, como el fundamento sólido sobre el que construir el futuro social y personal. No es una educación terminal sino dinamizadora y, por lo tanto, no pretende reducir o ajustarse a lo más imprescindible, sino posibilitar la continuidad del aprendizaje. La idea de mínimo, en la medida que implica un límite al derecho a la educación, está descartada de la visión ampliada que se quiere dar.

La educación básica no es un fin en sí misma (...).

La que satisface las necesidades fundamentales de la gente para que pueda sobrevivir, desarrollar sus propias capacidades, lograr una vida y un trabajo dignos, participar plenamente en el desarrollo local y nacional, mejorar la calidad de vida, tomar decisiones informadas y continuar aprendiendo (art. 1).

La educación de base no pretende adaptar a la supervivencia sino posibilitar la participación responsable y creativa en la sociedad. La educación básica es una fuerza, una posibilidad, y no un parche o un remedio. La educación básica es un fundamento que apunta hacia un techo, no es un tejado provisional. Este concepto se opone a la idea, generalizada entre los administradores de la educación básica de adultos, de que diseñar el currículum 
de adultos supone reducir o achicar el de la educación primaria escolar, y también la de los profesionales que consideran que dedicarse a la educación de adultos es una tarea más fácil y menos exigente que la de educar a niños.

Tampoco la educación básica ha de vincularse a la necesidad de resolver problemas inmediatos del medio local en el que se vive y cuyos aprendizajes se entiende que han de ser inmediatamente utilizables y aprovechables en la vida cotidiana. Este concepto de relevancia curricular que implica aprender lo que le exige y demanda su medio, cuando se hace de una manera exclusiva le adapta tanto a las circunstancias locales que impiden el aprendizaje crítico, el cual necesita una cierta distancia de las condiciones de vida inmediatas para poderlas reconstruir y transformar. La educación básica ligada exclusivamente a lo inmediato contiene todas las condiciones para reproducir las situaciones dadas y para integrarse, sin capacidad de reacción, en procesos sociales dominantes.

Resumimos en forma de esquema las diferencias entre el concepto de educación mínima y educación básica que se está generando en el campo de la educación de adultos:

\begin{tabular}{|c|l|}
\hline Educación minima & \multicolumn{1}{|c|}{ Educación básica } \\
\hline $\begin{array}{l}\text { Ofrece menos de lo mismo que se ofre- } \\
\text { ce en la educación escolar obligatoria. }\end{array}$ & Ofrece algo diferente. \\
\hline
\end{tabular}

Facilita el acceso al certificado de educación elemental.

Se intenta aligerar el aprendizaje, entendido como un peso.

No cuestiona el sistema escolar: se adapta a los adultos la enseñanza infantil primaria y la enseñanza juvenil universitaria.

Es un techo, un fin en sí misma. Pocos adultos continúan aprendiendo después de obtener el título mínimo.

Mira hacia la recuperación de oportunidades pasadas.

Posibilita seguir aprendiendo.

El aprendizaje es un valor que atrae.

Es un desafío para el sistema escolar aplicado a adultos que tiene que imaginar procesos de educación básica diferentes a los de la escuela primaria y procesos de educación continuada diferentes a los universitarios.

Es un cimiento, una puerta abierta. Pretende que los adultos sigan aprendiendo e investigando y facilita a los adultos su participación en la producción social del conocimiento y de la realidad social.

Pretende emprender un futuro.

\subsubsection{La educación básica ha de responder a las necesidades de los que aprenden y se centra sobre el aprendizaje}

Que el incremento de las posibilidades de educación se traduzca en un desarrollo genuino del individuo o de la sociedad depende, en 
definitiva, de que los individuos aprendan verdaderamente como resultado de esas posibilidades, esto es, de que verdaderamente adquieran conocimientos útiles, capacidad de raciocinio, aptitudes y valores (art. 4).

Partir y tener en cuenta la perspectiva de los que aprenden no implica más de lo mismo, es decir, fortalecer o cuidar el proceso de aprendizaje a través de estrategias y técnicas intraeducativas, sino que, además, incluye, al menos en la práctica educativa con personas adultas, otras dimensiones como las siguientes:

- Tener en cuenta las razones y motivaciones que el que quiere aprender se da o no se da para justificar su participación en los procesos de aprendizaje. La motivación real de los participantes es un factor esencial. No basta que haya razones sociales, políticas o económicas, no basta que los educadores las vean claras. Es necesario fijarse además en las razones antropológicas. Es fundamental escuchar las interpretaciones concretas que, sobre el aprendizaje, hacen los mismos que quieren aprender y descubrir el valor que dan al saber.

- La educación básica, desde la perspectiva de la atención al aprendizaje, ha de centrarse en un modelo de demanda y no siempre en un modelo de oferta. La demanda tiene en cuenta el punto de vista del que necesita formación (los adultos, la sociedad, los padres...) y no sólo la de los administradores educativos que imparten enseñanza. Es curioso, en este sentido, que un sistema de enseñanza tan contaminado en la sociedad actual por tantos elementos económicos esté siendo al mismo tiempo tan impermeable al dinamismo de la oferta y la demanda y que todavía siga predominando la escasez de pactos educativos entre administradores de la educación y administrados, entre profesores y alumnos, entre necesidades reales y ofertas académicas. Todavía sigue siendo dominante en el diseño de los procesos de aprendizaje el criterio del que ofrece, y pocos pasos se dan para que, en ese diseño, participe el que quiere aprender. Por supuesto no se está defendiendo la neoliberalización de la educación, sino la flexibilidad de la administración ante las necesidades educativas de las personas adultas.

- Desde la perspectiva del aprendizaje, se ha de tener en cuenta lo ya adquirido independientemente de dónde, cómo y cuándo se haya aprendido. Los aprendizajes no comienzan desde cero y desde la ignorancia absoluta. El que aprende es considerado habitualmente no desde lo que aporta y trae ya sabido, sino desde lo que no sabe o sabe mal según el criterio del educador. Valorar lo ya adquirido y considerar al participante en positivo, y no como "deficiente", es una de las consecuencias de esta atención al aprendizaje. 


\subsubsection{La educación básica no se desarrolla en un solo sistema sino en una red de aprendizaje}

Las necesidades básicas de aprendizaje de jóvenes y adultos son diversas y pueden satisfacerse mediante sistemas variados (art. 5).

El aprendizaje no se produce en situación de aislamiento. Los conocimientos y las capacidades para mejorar las condiciones de aprendizaje de los niños deben integrarse en los programas comunitarios de aprendizaje para adultos. La educación de los niños y la de sus padres se respaldan mutuamente y esta interacción debería aprovecharse para crear, en beneficio de todos, un ambiente de aprendizaje cálido y estimulante (art. 6).

Será necesaria la concertación de acciones entre todos los subsectores y todas las formas de educación, teniendo en cuenta el especial papel del profesional docente y de los administradores y demás personal de educación; la concertación de acciones entre el ministerio de educación y otros ministerios, entre ellos y los de planificación, hacienda, salud, trabajo, comunicación y otros sectores sociales; la cooperación entre organizaciones gubernamentales y no gubernamentales, el sector privado, las comunidades locales, los grupos religiosos y la familia (art. 7).

La necesidad de plantear la educación básica a través de una red implica, en los procesos educativos con personas adultas, las siguientes consecuencias:

- Los aprendizajes adquiridos, independientemente de cómo, dónde, cuándo y con quién se hayan aprendido, necesitan mecanismos de reconocimiento y validación social. La sociedad que centra la educación en un sistema educativo monopolista sólo valora y valida lo que ese sistema enseña, desperdiciando así muchos aprendizajes necesarios, sólo por el hecho de haber sido adquiridos fuera del propio sistema. Los aprendizajes, si son reales, pueden ser útiles para el mantenimiento de nuestra sociedad independientemente de la fuente de donde provengan. Si consideramos las habilidades no escolares sobre las que está soportada nuestra sociedad, nos sorprenderíamos a nosotros mismos. Son muchos los ejemplos concretos que se podrían poner: las habilidades que las madres de familia utilizan para cuidar a los hijos o a los mayores son en muchos casos las mismas que desarrolla una auxiliar de clínica o una enfermera, pero sin embargo no son reconocidas para poder ser utilizadas en un centro hospitalario sólo por el hecho de no haber sido adquiridas de la misma manera que se adquieren en el sistema escolar; lo mismo podríamos decir de habilidades comunicativas que aprenden los responsables sindicales o de asociaciones ciudadanas, que les permiten hablar, explicarse y pactar entre diferentes intereses. Son habilidades que tampoco suelen ser reconocidas socialmente porque no 
se han aprendido en la clase de lengua. Así podríamos enumerar muchas habilidades no escolares, pero fundamentales, que están sustentando el funcionamiento de nuestra sociedad y que necesitarían mecanismos ágiles de reconocimiento y validación.

- Los aprendizajes no dependen sólo de la enseñanza y del profesor. Se aprende en la vida, en las relaciones laborales, en el trabajo, en las relaciones cotidianas, en el desempeño de responsabilidades sociales, etc. Se aprende desde la pregunta y no sólo desde la respuesta. Se aprende en silencio. Si consideramos además que nuestra sociedad se caracteriza por no recluir el conocimiento en templos sagrados de difícil acceso y al amparo de celosos vigilantes, sino que circula y se publica por la red sin pedir permiso a nadie y sin pasar los filtros de selección de épocas anteriores, la importancia que adquiere el que aprende se acentúa aún más. En este sentido, el "aprendiz" adulto necesita intervenir cada vez más en la gestión de su aprendizaje. Antes el conocimiento coincidía con la información que daba el profesor, pero hoy existe tanta información que se necesita aprender a orientar la mirada y el oído para poder conocer. En un mundo donde las fuentes de información son tan plurales, necesita saber para informarse, porque "quien no sabe es como el que no ve", y necesita estructurar la información para conocer, porque "el poder no está en quien tiene la información" sino en el que sabe producir conocimientos con ella. Saborear y digerir los conocimientos que se van adquiriendo es la sabiduría que integra, además de la cabeza, el corazón y la mano; además de la razón, el sentimiento y el compromiso. Dimensiones que el hombre adulto no puede aplazar para un después ni delegar en otro.

- Existen sistemas paralelos de enseñanza con distinto reconocimiento social, y que solemos denominar sistema formal y sistema no formal. Lo válido y prestigiado suele ser lo aprendido en el sistema formal, lo aprendido en el sistema no formal es considerado como inferior, un aprendizaje de segunda categoría, sin el mismo reconocimiento social. Este tipo de paralelismo entre sistemas de primera y segunda debería ser conectado a través de pasarelas reales y flexibles que hicieran del aprendizaje un verdadero contínuum espacial o institucional que, además de permitir un tránsito sin dificultades burocráticas entre los distintos sistemas, instituciones y modalidades de enseñanza, diluyera las desigualdades entre aprendizajes sólo por su diferente procedencia. Para ello se necesita también un mecanismo de confianza entre las distintas instituciones y sistemas.

- El aprendizaje en red implica también un aprendizaje dialógico que rompa la dinámica de relación entre experto e ignorante. Todos aprendemos de todos. En muchos campos y áreas, el conocimiento puede construirse socialmente dialogando y compartiendo, sin confundir los roles y las funciones de cada participante, pero sin anular a nadie. No esta- 
mos defendiendo con esta postura la trivialización del científico en la construcción del saber, sino su necesaria modestia para aprender a ver desde otra perspectiva, también necesaria en la construcción de un saber más objetivo.

\subsubsection{La educación básica ha de realizarse en un contexto intersectorial}

Este tratamiento es sin duda el más profundo y difícil de poner en práctica y donde se alargan más las distancias entre lo que se dice y lo que se hace, entre la teoría y la práctica, entre las declaraciones y los procesos concretos. Lo que se viene a plantear desde esta perspectiva es que los grandes problemas de la educación y, en concreto, de la alfabetización y educación básica para todos no se resuelven única y exclusivamente desde la pedagogía sino que otros sectores de la realidad y de la ciencia han de intervenir también en su solución. Las implicaciones de un tratamiento intersectorial de la educación básica apuntan hacia las siguiente direcciones:

Desarrollar políticas de apoyo en los sectores social, cultural y económico para poder impartir y aprovechar de manera cabal la educación básica con vistas al mejoramiento del individuo y de la sociedad (art. 8).

Dispensar educación básica a todos depende de un compromiso y una voluntad politicos apoyados en adecuadas medidas fiscales (art. 8.1).

La sociedad debe proporcionar además un sólido ambiente intelectual y científico a la educación básica (art. 8.2).

La satisfacción de las necesidades básicas de aprendizaje constituye una común y universal tarea humana. Para llevar a cabo esa tarea se requieren la solidaridad internacional y unas relaciones económicas justas y equitativas a fin de corregir las actuales disparidades económicas (art. 10.1).

La calidad de la educación básica no depende sólo de mejorar las condiciones internas del proceso de aprendizaje tales como la de los edificios, los textos y manuales escolares, las bibliotecas, la capacitación de los docentes, la motivación de los educandos, facilitar incluso las posibilidades de estudio mediante becas, permisos de trabajo para el estudio, etc. La calidad educativa y los resultados del aprendizaje no dependen solamente de facilitar las condiciones intraescolares sino también, y quizás sobre todo, de factores sociales, culturales, económicos y políticos como el desempleo, la pobreza, la falta de acceso igualitaria a la información, a material de lectura y a internet, 
la falta de tiempo de determinados grupos sociales para el estudio y la reflexión, la falta de integración de muchos ciudadanos o la pobre integración en redes de relación social, cultural, sindical o política. Desde esta perspectiva, hablar de una educación básica intersectorial implica:

- La educación no se desarrolla sólo en el estricto ámbito de la enseñanza, en los demás sectores de la vida también hay que intervenir educativamente.

No es suficiente aprovechar por parte del sector educativo las posibilidades educadoras de los medios y las tecnologías de la información y la comunicación. Es necesario además que esos medios actúen más educativamente en su propio sector de actividad. A las nuevas tecnologías, por ejemplo, no sólo hay que traerlas a los medios intraeducativos o intraescolares para mejorar la escuela (enseñanza a distancia, internet en la escuela, videos educativos, etc.), sino que han de procurar mejorar la educación desde su actuación cotidiana extraescolar, en el sector de la comunicación, haciendo una televisión más educativa, facilitando el uso de redes de información, etc. Lo mismo puede ocurrir con la economía. Su misión educativa no consiste sólo en intervenir en el sector intraeducativo mediante becas o ayudas al estudio, sino haciendo posible, en las redes de producción, el ejercicio de todos los aprendizajes. Qué sentido puede tener para determinados sectores de población el aprendizaje, si en su universo de vida lo que se aprende no vale para casi nada. Qué sentido puede tener el aprendizaje cuando, en un contexto dominado por la economía, queda reducido a instrumento cuya utilización depende no del desarrollo humano, sino de las ganancias que genera. También podemos utilizar el ejemplo de la política en el mismo sentido que lo hemos hecho con la economía. Si se estima, por ejemplo, que la cifra de la educación primaria para todos equivale a 8000 millones de dólares adicionales cada año, y esta cifra equivale a los gastos con fines militares durante cuatro días, es claro que la voluntad política internacional de intervenir en la decisión de afrontar la educación para todos no puede quedar recluida en decisiones escolares, al margen de determinadas decisiones sobre el reparto general del gasto público.

- Los distintos profesionales no han de centrar su profesionalidad sólo en las cuestiones técnicas, sino también en cuestiones humanitarias.

Desde esta perspectiva, habría que desbloquear tanto aquellas conductas profesionales que no aciertan a ver el bosque en medio de tanto árbol como aquellas que no aciertan a ver el árbol en medio de tanto bosque. Una relación más estrecha entre lo macro y lo micro aparece cada vez más necesaria entre los profesionales. Es fácil encontrar grandes analistas del paro que no terminan de vislumbrar al parado, o profesionales de la sanidad que están tan centrados en las enfermedades que no se pueden permitir el lujo de conversar con los enfermos. Pero también encontramos a profesionales perdidos en los sentimientos de compasión hacia los parados o los enfermos que no saben situar 
los problemas en marcos estructurales. Los profesionales de la educación también están divididos entre aquéllos que se centran en la microenseñanza que conduce a la mejora de los procesos dentro del aula y la macroenseñanza que conduce a la mejora de la enseñanza desde fuera del aula. Mientras en la microenseñanza dominan educadores y pedagogos, en la macroenseñanza dominan economistas, sociólogos, filósofos, políticos. Un diálogo e interacción entre ambas perspectivas parece inevitable si no queremos convertir a los microeducadores en miopes ante los problemas de fondo y a los macroeducadores en insensibles ante los procesos y necesidades concretas.

- Los mismos educandos deben situarse en una dimensión intersectorial, reconociendo que el aprendizaje no es sólo un instrumento para promocionar académicamente o para ser utilizado en un determinado modelo de producción de la sociedad del conocimiento, sino que también el aprender es un valor antropológico y un constitutivo de una vida mejor y más autónoma que permite disfrutar de las distintas perspectivas culturales que nos ofrece un mundo tan variado y pluricultural.

\section{EVALUACIÓN DE LA EPT EN EL FORO DE DAKAR}

Durante esta década han existido cambios positivos en la educación de base: se ha aumentado el número de matrículas en primaria en unos 82 millones. A pesar de que el analfabetismo sigue teniendo proporciones inaceptables, se han logrado algunos avances: el índice de alfabetización de adultos pasó a un $85 \%$ en los hombres y a un $74 \%$ en las mujeres. Sin embargo los síntomas de retroceso hacia la visión más recortada de la educación frente a la visión ampliada de Jomtien que ya aparecieron en la conferencia de Amman (1995) ${ }^{6}$ se han vuelto a agudizar en Dakar.

La evaluación de Dakar sobre esta década ha demostrado un cierto retroceso en la aplicación del concepto ampliado de alfabetización y de educación. Se ha descuidado también el elemento de universalizar la educación a todos, reduciendo el "todos" a la educación primaria de niños y niñas. La educación básica de adultos, como dice M. R. Torres (2000: 26), se ha feminizado y rejuvenecido. Feminizado porque se ha centrado en el público femenino más marginado tradicionalmente, y rejuvenecido porque se ha privilegiado

- En Amman (1996) se hace una revisión del proceso seguido por el Programa de la Educación para Todos en la mitad de su recorrido. Allí Mayor Zaragoza, entonces Director de la UNESCO, observó que se estaba dedicando todo el esfuerzo a la enseñanza infantil, a posibilitar el acceso a un puesto escolar y a un mayor tiempo de permanencia en la escuela, pero se estaba descuidando la educación de adultos y la atención a las nuevas dimensiones que implicaba el concepto amplio de educación básica que se había desarrollado en Jomtien. 
a los adultos de 14 a 24 años. Con ello la educación básica ha adquirido un sentido de compensación de la escuela primaria o de reparación defectuosa en el proceso de inserción social, cuando en el concepto ampliado de Jomtien tiene como objetivo crear fundamentos sólidos para participar activamente y construir o reconstruir críticamente el contexto social en el que se vive.

En el informe de la sección tercera de la evaluación del proceso del Foro Mundial sobre educación (www.unesco.org) se transcriben las intervenciones de algunos participantes, indicando el marcado retroceso de ciertos patrocinadores con relación a la visión ampliada original y las implicaciones de este retroceso sobre el trabajo del Foro (traducción personal del francés):

"Es un punto de exactitud histórica recordar que el Banco Mundial y la UNICEF, los dos, han reducido la noción de la EPT de Jomtien, más o menos inmediatamente después de Jomtien, para centrarse exclusivamente sobre la escolaridad primaria. Se trataba únicamente de una comodidad corporativa. La educación no formal y la alfabetización eran consideradas demasiado modestas, cuyos progresos son difíciles de evaluar, demasiado frágiles administrativamente para absorber el volumen importante de financiación de las agencias, demasiado débiles como prioridad política. La UNESCO es la única agencia especializada en educación no formal y educación de adultos, apoyada por el PNUD convencido de la importancia de la formación de base en relación con el mundo del trabajo. El FNUAP ha seguido detrás con su concepto de educación de la población y ha añadido a su agenda la educación de las mujeres".

"Los convocantes han incumplido sus compromisos. La educación no formal de niños y adultos ha sido globalmente descuidada, aunque el Banco Mundial y UNICEF hayan hecho esfuerzos. La educación de Hamburgo de la UNESCO ha sido una buena cosa. Ha permitido poner carne sobre el esqueleto. El Forum debería haber recogido las ideas de esta conferencia. En lugar de esto, se ha reducido al sistema educativo formal".

"La UNESCO ha preconizado una concepción abierta de la educación de adultos. La UNICEF ha subrayado la necesidad de incluir en la educación de base el desarrollo de la infancia y la educación precoz. La UNICEF y la UNESCO han defendido las dos la necesidad de introducir modalidades educativas flexibles y diversificadas, incluyendo alternativas educativas no formales. El Banco Mundial quería centrarse sobre el sistema escolar y la educación primaria. El PNUD no ha tomado postura sobre este punto".

Efectivamente, a lo largo del proceso, ha existido una tensión oculta entre los mismos patrocinadores por no asumir en la práctica la visión teórica que apareció en la declaración de 1990. El desacuerdo entre UNESCO y UNICEF 
sobre la puesta en marcha concreta del programa de educación para todos ha facilitado que la política educativa del Banco Mundial ${ }^{7}$, menos especializado en educación, haya sido la que haya prevalecido, favoreciendo por una parte un concepto más recortado de la educación y por otra parte una contaminación del comportamiento del sector económico en el educativo. Si en Jomtien se hablaba de educación para todos dentro y fuera del sistema escolar, lo que se ha puesto en marcha a lo largo de la década ha sido sobre todo la educación de la infancia. Si lo que se planteaba era satisfacer necesidades básicas de aprendizaje, lo que se potencia en realidad es la enseñanza primaria. Si lo que se planteaba en Jomtien era aprendizaje de calidad, lo que se considera en Dakar es el acceso a un puesto de aprendizaje en la escuela independientemente de sus resultados y, si la equidad era un objetivo claro, durante esta década se produce un aumento de la pobreza, la exclusión, el desempleo y el hambre. Por otra parte, siendo verdad que en los años noventa aumenta la asistencia internacional asignada a la educación básica, se produce sin embargo una disminución de la ayuda total al desarrollo ${ }^{8}$. El planteamiento intersectorial de la educación a desarrollar con una estrategia de redes educativas sigue dominado todavía por la persistencia de sistemas férreos especializados en la enseñanza que se resisten a perder su monopolio. No deberíamos olvidar un detalle de no menor importancia y es que en Dakar no estuvieron juntos en el mismo Foro los representantes oficiales y los de las ONGs. Además del Foro oficial hubo el "foro alternativo", cosa, por ejemplo, que ya en la Conferencia Internacional sobre educación de Adultos, celebrada en 1997 en Hamburgo, no se produjo, en nombre precisamente del principio dialógico proclamado en Jomtien?. Por otra parte, el avance cuan-

${ }^{7}$ La noción de educación básica del Banco Mundial durante esta década sigue centrada en la educación escolar e infantil, como se puede demostrar a partir de las definiciones que ofrece en los distintos documentos a lo largo de esta década. En Educación primaria. Documento de Politica del Banco Mundial. Washington de 1992, identifica la enseñanza básica con la primaria. En Prioridades y estrategias para la educación: examen del banco mundial. Washington de 1996, la definición de educación básica varía según el país, aunque normalmente, se dice, abarca por lo menos la educación primaria y con frecuencia también la secundaria de primer ciclo (BM 1996:117) El BM no considera otras esferas de lo educativo como la familia, la comunidad, el entorno, el trabajo, los medios de comunicación que quedan al margen de las recomendaciones de su política. También queda fuera la educación de adultos y la educación no formal. El BM fomenta así una política intraescolar centrada en edificios (política que cambia en 1991), bibliotecas, libros de textos, tecnología aplicada a la escuela con el fin de mejorar la oferta escolar, pero no una política educativa extraescolar y de demanda, fomentando de esta manera una visión recortada de la educación diferente de la visión ampliada tal y como firmó en Jomtien con el resto de Organismos patrocinadores de la Conferencia sobre la Educación para todos. Ver el análisis de R. M. Torres 1997): ¿Mejorar la calidad de la educación básica? Las estrategias del Banco Mundial. Editorial CEM. Buenos Aires.

${ }^{8}$ Comentario detallado del Marco de acción de Dakar. Documento preparado por el Comité de redacción del Foro Mundial sobre educación a partir de las sugerencias presentadas antes y durante el foro mundial y, en particular, las formuladas en las 24 reuniones sobre estrategia. París, 23 de mayo de 2000.

${ }^{9}$ El Foro alternativo o consulta Internacional de las ONGs se celebró el 24 y 25 de abril, dos días antes del foro oficial. 
titativo contra la desaparición del analfabetismo no ha sido reducido, a pesar del esfuerzo alfabetizador que anteriormente hemos indicado. Si en 1990 había 895 millones de analfabetos adultos, en el 2000 quedan 880 millones, y el objetivo de reducir en un $50 \%$ el analfabetismo adulto se vuelve a aplazar de nuevo para el año 2015. Los indicadores utilizados finalmente, en el foro de Dakar, para evaluar la década son un signo evidente de este retroceso.

\subsection{LOS INDICADORES DE EVALUACIÓN, UN SÍNTOMA DE RETROCESO}

Si nos atenemos a los indicadores de evaluación utilizados en Dakar para evaluar las tareas y el concepto de educación, hemos de decir que no son los más adecuados y que responden más a la medición de una tarea educativa según la visión restringida, tradicional y escolar, que a la visión ampliada propuesta en Jomtien.

Indicadores de base para evaluar una década de "educación de adultos"

Indicador 1 Tasa bruta de matrícula en programas de desarrollo de la primera infancia, en el sector público, privado y comunitario, expresada en porcentaje del grupo de edad oficial correspondiente, o dentro del grupo de edad oficial comprendido entre los 3 y los 5 años.

Indicador 2 Porcentaje de nuevos alumnos que ingresan al primer grado de la enseñanza primaria, a quienes se ha ofrecido un programa organizado de desarrollo de la primera infancia.

Indicador 3 Tasa bruta de matrícula: nuevos alumnos que ingresan al primer grado de enseñanza primaria como porcentaje de la población en edad oficial de ser admitida en este nivel de enseñanza.

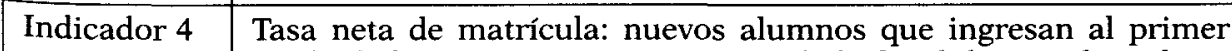
grado de la enseñanza primaria en edad oficial de ser admitidos a este nivel como porcentaje de la población correspondiente.

\begin{tabular}{|l|l|}
\hline Indicador 5 & Tasa bruta de escolarización. \\
\hline Indicador 6 & Tasa neta de escolarización. \\
\hline Indicador 7 & $\begin{array}{l}\text { Gasto público ordinario destinado a la enseñanza primaria: (a) en } \\
\text { porcentaje de PIB y (b) por alumno, en porcentaje de PIB por habi- } \\
\text { tante. }\end{array}$ \\
\hline Indicador 8 & $\begin{array}{l}\text { Gasto público destinado a la enseñanza primaria en porcentaje de } \\
\text { total de gasto público en educación. }\end{array}$ \\
\hline Indicador 9 & $\begin{array}{l}\text { Porcentaje de docentes de enseñanza primaria que poseen título } \\
\text { académico requerido. }\end{array}$ \\
\hline Indicador 10 & $\begin{array}{l}\text { Porcentaje de docentes de enseñanza primaria acreditados para la } \\
\text { enseñanza de acuerdo a las normas nacionales. }\end{array}$ \\
\hline
\end{tabular}


Indicadores de base para evaluar una década de "educación de adultos" (Continuación)

\begin{tabular}{|c|c|}
\hline Indicador 11 & Número de alumnos por docente. \\
\hline Indicador 12 & Tasa de repetición por grado. \\
\hline Indicador 13 & $\begin{array}{l}\text { Tasa de supervivencia en el quinto grado de escuela primaria (por- } \\
\text { centaje de cohorte que alcanza efectivamente el quinto año). }\end{array}$ \\
\hline Indicador 14 & $\begin{array}{l}\text { Coeficiente de eficacia (número ideal de años-alumnos necesarios } \\
\text { para que una cohorte complete el ciclo primario, expresado en por- } \\
\text { centaje del número efectivo de años-alumnos). }\end{array}$ \\
\hline Indicador 15 & $\begin{array}{l}\text { Porcentaje de alumnos que han alcanzado como mínimo el cuarto } \\
\text { grado de primaria y que dominan un conjunto de competencias } \\
\text { básicas definidas a escala nacional. }\end{array}$ \\
\hline Indicador 16 & Tasa de alfabetización de las personas entre 15 y 24 años. \\
\hline Indicador 17 & $\begin{array}{l}\text { Tasa de alfabetización de adultos: el porcentaje de la población de } \\
\text { más de } 15 \text { años que sabe leer y escribir. }\end{array}$ \\
\hline Indicador 18 & $\begin{array}{l}\text { Indice de paridad de alfabetización entre los sexos: relación entre } \\
\text { tasa de alfabetización de las mujeres y la de los hombres. }\end{array}$ \\
\hline
\end{tabular}

Fuente: Foro consultivo Internacional sobre EPT, Evaluación para Todos: evaluación en el año 2000. Directivas técnicas. UNESCO. París. 1998.

Efectivamente, si nos fijamos bien en los criterios elaborados para evaluar la EPT en la década por el Foro Consultivo, observaremos que la gran mayoría de los indicadores, quince de dieciocho, pretende medir la educación infantil y en todo caso la escolarización en primaria, con lo cual la filosofía de Jomtien que insistía por igual en la educación infantil y de adultos, desarrollada en la escuela o fuera de la escuela, queda desequilibrada. Además en los indicadores se insiste mucho en medir la tasa de matrícula, cuando en Jomtien se insistió (artículo 4) en "que la educación básica debe centrarse en las adquisiciones y los resultados efectivos del aprendizaje, en vez de prestar exclusivamente atención al hecho de matricularse". Por otra parte, los indicadores reservados para evaluar la alfabetización de mayores de quince años reducen el concepto de alfabetización (indicador 17) al hecho de saber leer y escribir, cuando en la declaración de Jomtien la capacidad de saber leer y escribir era uno de los requisitos para estar alfabetizados, pero no el único. La amplitud de las necesidades básicas de aprendizaje y la manera de satisfacerlas, se decía, varían según cada país y cada cultura y cambian inevitablemente con el transcurso del tiempo.

Podemos afirmar, por lo tanto, que se han producido dos lecturas diferentes de la declaración de Jomtien que han marcado procesos incoherentes con la declaración que allí se hizo hace diez años. 


\subsection{LOS RESULTADOS EN EDUCACIÓN DE ADULTOS DURANTE LA DÉCADA DE LOS NOVENTA VISTOS DESDE EL ANÁLISIS DEL INFORME MUNDIAL 2000 DE LA UNESCO SOBRE LA EDUCACIÓN DEL AÑO ${ }^{10}$}

El número estimado de analfabetos mayores de quince años ha aumentado en el mundo desde 1950 al año 2000 en aproximadamente 170 millones: en 1950 eran 705 millones y en el 2000 alcanzan la cifra de 875 .

Evolución del analfabetismo adulto de 1950 al 2000 en el mundo

\begin{tabular}{|l|c|c|c|}
\hline & 1950 & 1970 & 2000 \\
\hline Numero en millones & 705 & 858 & 875 \\
\hline Tasa & 49 & 37 & 21 \\
\hline Aumento de alfabetizados & & & 1926 millones más \\
\hline Aumento de analfabetos & & & 17 millones más \\
\hline
\end{tabular}

Evolución de las tasas de alfabetización de adultos por regiones

\begin{tabular}{|l|c|c|c|c|c|c|c|}
\hline & $\begin{array}{c}\text { Regiones } \\
\text { desarrolladas }\end{array}$ & $\begin{array}{c}\text { Africa } \\
\text { Subhariana }\end{array}$ & $\begin{array}{c}\text { Estados } \\
\text { Arabes }\end{array}$ & $\begin{array}{c}\text { América } \\
\text { Latina } \\
\text { Caribe }\end{array}$ & $\begin{array}{c}\text { Asia } \\
\text { Este }\end{array}$ & $\begin{array}{c}\text { Asia } \\
\text { Sur }\end{array}$ & $\begin{array}{c}\text { Paises } \\
\text { menos } \\
\text { desarrollados }\end{array}$ \\
\hline Año 1970 & 95 & 29 & 29 & 74 & 56 & 32 & 27 \\
\hline Año 2000 & 99 & 61 & 62 & 88 & 86 & 56 & 51 \\
\hline
\end{tabular}

\subsection{LOS ANÁLISIS CRÍTICOS SOBRE LA DÉCADA EDUCATIVA}

Quizás el pronunciamiento latinoamericano que tuvo lugar en el mismo contexto de Dakar pueda ser revelador de una cierta preocupación en el ámbito de los educadores sobre los resultados educativos de la década. Solamente transcribimos un párrafo para animar al lector a completar su lectura:

${ }^{10}$ Rapport mondial sur l'éducation 2000. Le droit à l'éducation. Vers l'éducation pour tous, tout au long de la vie. Editions UNESCO. p. 17. 
Nos preocupa enormemente la situación y el rumbo de la educación en el mundo, en los países en desarrollo y en nuestra región en particular. Después de varias décadas de intentos reiterados de reforma educativa en nuestros países, los resultados son dudosos y, en todo caso, no están a la vista en el ámbito que finalmente importa y que es el objetivo mismo de la educación: el aprendizaje y la formación integral de la persona. "Enfatizar el aprendizaje" fue un mandato fundamental para la Educación para Todos; no obstante, el indicador de aprendizaje (uno de los 18 indicadores que debían reportar los países) debió ser eliminado del informe final de evaluación de la década pues la mayoría de países no tenía información para reportar. "Mejorar el aprendizaje" fue la consigna de la década de 1990 en nuestra región; no obstante, las evaluaciones de rendimiento escolar hechas en los últimos años en los diferentes países muestran resultados pobres, por debajo de lo esperado y de lo deseable. Tampoco cuentan los sistemas educativos con indicadores o evidencias de logro en torno a la formación de los educandos, entendida como despliegue de sus potencialidades, estímulo a su creatividad y consolidación de sus valores ${ }^{11}$.

Otros analistas resumen la labor educativa de esta década en forma de distancia entre la propuesta optimista de Jomtien y la respuesta obtenida:

\begin{tabular}{|l|l|}
\hline \multicolumn{1}{|c|}{ Propuesta } & \multicolumn{1}{|c|}{ Respuesta } \\
\hline Educación para todos. & $\begin{array}{l}\text { Educación para niños y niñas (los más } \\
\text { pobres entre los más pobres). }\end{array}$ \\
\hline $\begin{array}{l}\text { Educación básica. } \\
\text { Universalizar la educación básica. }\end{array}$ & $\begin{array}{l}\text { Educación escolar (primaria). } \\
\text { Universalizar el acceso a la educación } \\
\text { primaria. }\end{array}$ \\
\hline Necesidades básicas de aprendizaje. & Necesidades mínimas de aprendizaje. \\
\hline Concentrar la atención en el aprendizaje. & Mejorar y evaluar el rendimiento escolar. \\
\hline Ampliar la visión de la educación básica. & $\begin{array}{l}\text { Ampliar el tiempo (número de años) de } \\
\text { la escolaridad obligatoria. }\end{array}$ \\
\hline $\begin{array}{l}\text { Educación básica como cimiento de de } \\
\text { aprendizajes posteriores. }\end{array}$ & $\begin{array}{l}\text { Educación básica como un fin en sí } \\
\text { misma. }\end{array}$ \\
\hline Mejorar condiciones de aprendizaje. & $\begin{array}{l}\text { Mejorar condiciones internas de la ins- } \\
\text { titución escolar. }\end{array}$ \\
\hline Todos los países. & Los países en desarrollo. \\
\hline $\begin{array}{l}\text { Responsabilidad de los países (organis- } \\
\text { mos gubernamentales y no-guberna- } \\
\text { mentales) y la comunidad internacional. }\end{array}$ & Responsabilidad de los países. \\
\hline
\end{tabular}

Fuente: Sacado de R.M. Torres (2000): Una década de educación para todos: La tarea pendiente. IIPE. UNESCO. Buenos Aires

${ }^{11}$ Ver dirección electrónica http:/WWW.unesco.org 


\section{Para terminar, la perspectiva optimista del director general de la UNESCO}

Si la decisión de aplazar hasta el año 2015 la fecha para cumplir los objetivos de Jomtien supone un retraso de 15 años injustificable para muchos colectivos, para otros, en cambio, supone el plazo de tiempo para un esfuerzo más realista y exigente. A estos últimos, Koichiro Matsuura, Director General de la UNESCO, les ofrece unas perspectivas de compromiso más crítico pero no menos optimista. Transcribimos algunas palabras de su discurso ante el Foro de Dakar:

El Foro de Dakar... su balance nos ha obligado a pasar sin más demora a la autocrítica. Pues si el inventario mundial de la educación para todos muestra que estos diez años se han llevado a cabo sustanciales progresos en el campo educativo (la escolarización primaria ha ido incrementándose a un ritmo de 10 millones de niños más anualmente $y$, dato muy importante y esperanzador, la tasa de escolarización aumenta ahora más rápidamente que el crecimiento demográfico), por el contrario, no podemos estar satisfechos ni autocomplacernos de que en el año 2000 aún permanezcan alrededor de 113 millones de niños sin escolarizar y 875 millones de analfabetos.

La importancia de la reunión de Dakar, tras la detallada exposición de aciertos y errores de las políticas educativas del decenio, emana también, y sobre todo, del marco de acción en ella adoptado. La educación, definida como un derecho humano fundamental y la clave para el desarrollo sostenible y la paz, se plasma en marco de acción para quince años. En él, los alli participantes nos comprometemos sustancialmente a asegurar que para el 2015 todos los niños tengan acceso a una educación primaria obligatoria, completamente gratis y de buena calidad; a conseguir el 50\% de aumento de alfabetización de adultos, así como eliminar la diferencia de géneros en la educación primaria y secundaria para el 2005 y lograr la equidad completa entre el hombre y la mujer para el 2015.

El esfuerzo principal de esta tarea incumbe, naturalmente, a cada país, ya que alrededor del $63 \%$ del coste total de la educación en el mundo corre a cargo de los estados, el $35 \%$ de los fondos privados (padres, alumnos, comunidades, empresas, ONGs) y el $2 \%$ proceden de programas internacionales de ayuda al desarrollo.

Se estima que la realización de estos objetivos necesitará un esfuerzo financiero suplementario de los países, asi como de los donantes bilaterales y multilaterales, de aproximadamente 8.000 millones de dólares anuales durante un decenio para lo que es preciso un firme compromiso de los gobiernos nacionales, de las institu- 
ciones donantes, inclusive del Banco Mundial, así como de los bancos Regionales de Desarrollo.

La Organización de las Naciones Unidas para la Educación, la Ciencia y la Cultura (UNESCO), institución internacional cuya misión por excelencia es precisamente la defensa de la causa educativa en el mundo, asumirá la secretaría, manteniendo su papel de coordinadora y propiciando una auténtica dinámica de cooperación entre los socios de la Educación para Todos.

\section{BIBLIOGRAFÍA}

Alfabetización para todos: una visión renovada para un plan decenal de acción. Participación de la División de educación básica de la UNESCO. Documento Dakar 2000.

Banco Mundial: Prioridades y estrategias para la educación: examen del Banco Mundial. Washington, 1996.

Banco Mundial: Educación primaria. Documento de Politica del Banco Mundial. Washington de 1992.

Comentario detallado del Marco de acción de Dakar. Documento preparado por el Comité de redacción del Foro Mundial sobre educación a partir de las sugerencias presentadas antes y durante el foro mundial y, en particular, las formuladas en las 24 reuniones sobre estrategia. París, 23 de mayo de 2000.

Foro consultivo Internacional sobre EPT, Evaluación para Todos: evaluación en el año 2000. Directivas técnicas. UNESCO. París. 1998.

Forum Mondial sur l'éducation: Le processus. http://www2.unesco,orgwef/ fr-leadup/fr rmeet europ doctrav. shtm

Marco de acción para satisfacer las necesidades básicas de aprendizaje. Direc- trices para poner en práctica la Declaración Mundial sobre educación para todos. Jomtien 1990.

Marco de Acción de Dakar: Educación para todos: cumplir nuestros compromisos comunes. Texto aprobado por el Foro Mundial sobre Educación. Dakar. Senegal, 26-28 de abril de 2000.

ORDÓNEZ, V.: Las lecciones de una déca$d a$ en FUENTES-UNESCO. Abril $2000, \mathrm{n}^{\circ} 122$.

TORRES, R.M. (1997): ¿Mejorar la calidad de la educación básica? Las estrategias del Banco Mundial. Editorial CEM. Buenos Aires.

TORRES, R.M. (2000): Una década de educación para todos: La tarea pendiente. IIPE. UNESCO. Buenos Aires.

UNESCO: Cinquième Conférence Internationales sur l'éducation des adultes (Confintea V): Agenda pour l'avenir. Hambourg, 14-18 Juillet 1997.

UNESCO: Educación para todos. Diez años después de Jomtien. FUENTES. $\mathrm{N}^{\circ}$ 122. Abril 2000.

UNESCO: Rapport mondial sur l'éducation 2000. Le droit à l'éducation. Vers l'éducation pour tous, tout au long de la vie. Editions UNESCO. 


\title{
RESUMEN
}

El contenido que aparece en este articulo hace referencia a la evolución que ha tenido lugar en la educación de adultos durante la década de los años noventa. El marco de referencia es la conferencia de Jomtien en 1990 y el Foro Mundial de la Educación celebrado en abril del año 2000 para evaluar el plan de acción elaborado en Jomtien.

Después de revisar lo que en 1990 suponía el concepto ampliado de la educación, se constatan ciertos signos de retroceso en su aplicación y en el incumplimiento del programa. Se analizan algunos datos del informe mundial sobre la educación publicado por la UNESCO en el año 2000 y se tienen en cuenta algunos análisis de especialistas y participantes en el Foro de Dakar que corroboran este retroceso. Se quiere terminar con unas perspectivas optimistas para el año 2015 ofrecidas por el actual director General de la UNESCO.

Palabras clave: Conferencia de Jomtien, Foro de Dakar, concepto ampliado de educación, informe mundial de la educación 2000

\begin{abstract}
This article discusses the development of adult education during the 1990s. The frame of reference is the Jomtien Conference in 1990 and the World Education Forum held in April 2000 to evaluate the plan of action drawn up at Jomtien. After reviewing what, in 1990, constituted the concept of education in the wider sense, certain signs of non-fulfilment and of retrogresion in the application of the program are highlighted. Data from the World Report on Education published by UNESCO in the year 2000 is analysed, and the analysis of specialists and participants in the Dakar Forum which corroborate this retrogresion are also discussed. The article concludes, nevertheless, with some optimistic perspectives offered by the present Director General of UNESCO.
\end{abstract}

Key words: Jomtien Conference, Dakar Forum, concept of Education in the wider sense, World Report on Education 2000. 